\title{
Improvement of Vitiligo after Concurrent Treatment of Hypothyroidism: A Case Report
}

\author{
Tasneem Mohammad ${ }^{1}$, Evelyn Sommariva C $^{2}$, Angela Parks-Miller ${ }^{1,2}$ and Iltefat Hamzavi ${ }^{1,2 *}$
}

${ }^{1}$ Department of Dermatology, Henry Ford Hospital, Detroit, Michigan, USA

${ }^{2}$ Dermatology Specialists of Canton, Canton, Michigan, USA

\begin{abstract}
Vitiligo is a disorder of pigmentation characterized by the development of depigmented macules and patches. The etiology is multifactorial, including immune mediated destruction of melanotytes. Several autoimmune conditions are associated with vitiligo, but thyroid disease is the most common. In this case report, we describe a patient with rapidly depigmenting vitiligo who stabilized on narrowband UVB (NBUVB) phototherapy and oral corticosteroids, and began showing dramatic repigmentation once thyroid replacement was initiated. This highlights the interplay between vitiligo and autoimmune thyroid disease, as well as the need for adequate treatment of both disorders to achieve optimal treatment response.
\end{abstract}

Keywords: Vitiligo; Hypothyroidism; Treatment; Autoimmune; Phototherapy

\section{Introduction}

Vitiligo is a disorder of pigmentation characterized by the development of depigmented macules and patches over the body. It affects approximately $0.5-1 \%$ of the population worldwide and is associated with significant psychosocial impact [1]. The etiology of vitiligo is multifactorial, including an impaired ability to metabolize reactive oxidative species (ROS) as well as the immune mediated destruction of melanocytes [2]. It has been noted that patients with vitiligo are at increased risk of developing other autoimmune diseases including hypothyroidism, alopecia areata, and Type I Diabetes Mellitus (DM). In fact, thyroid dysfunction in people with vitiligo is approximately $20-30 \%$, which is significantly greater than the general population [3-5]. In this case report, we highlight the case of a patient with rapidly depigmenting vitiligo and concurrent hypothyroidism who experienced significant repigmentation after initiating thyroid supplementation coupled with phototherapy.

\section{Case Report}

A 25 year old Caucasian female with skin phototype III and rapidly depigmenting generalized vitiligo presented to clinic with greater than $75 \%$ of body surface area involvement. Her family history was significant for vitiligo in her maternal great-grandmother and psoriasis in both her mother and brother. Other autoimmune conditions, including thyroid dysfunction in her maternal grandmother and mother and Type I DM in her maternal grandmother, were also noted at the initial encounter. Her review of systems was remarkable for fatigue and weight gain, but she denied any palpitations, cold intolerance, dry skin, menstrual irregularities, or muscle weakness. She was started on prednisone $20 \mathrm{mg}$ daily and narrowband UVB phototherapy for treatment of vitiligo. Thyroid function labs were drawn to rule out potential thyroid dysfunction. At one month followup, despite no significant repigmentation, stability of disease was achieved and she was continued on phototherapy, with tapering of prednisone to every other day dosing. TSH levels drawn at the initial visit came back elevated at $7.59 \mu \mathrm{IU} / \mathrm{mL}$ (normal 0.34-3.00 $\mu \mathrm{IU} / \mathrm{mL}$ ) with a T4 in the low normal range at $0.83 \mathrm{ng} / \mathrm{dL}$ (normal $0.61-1.35 \mathrm{ng}$ / $\mathrm{dL}$ ). Thyroid replacement was started by her primary care physician as the patient was symptomatic. Shortly thereafter, the patient began to note repigmentation of affected areas. Her oral prednisone was switched to pulse dosing of dexamethasone and an oral antioxidant was added in addition to continuing phototherapy. Approximately 3 months after treatment was started, depigmented lesions had achieved $50-90 \%$ overall re-pigmentation in a predominantly follicular pattern (Figure 1). Now completing the fourth month of treatment, the patient continues to note improvement in pigmentation.
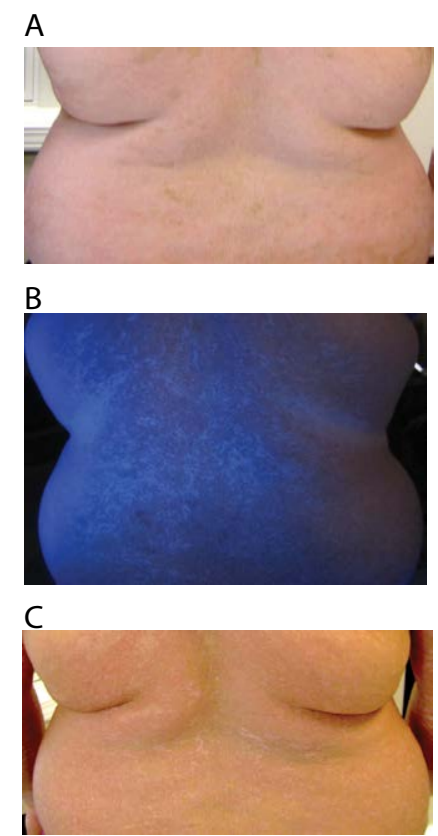

Figure 1: Patient photographs at baseline.

(1A) Back showing $>90 \%$ depigmentation

(1B) Back showing $>75 \%$ repigmentation in a predominantly follicular pattern

(1C) Back showing $>90 \%$ repigmentation in a predominantly follicular pattern

*Corresponding author: Iltefat Hamzavi, Department of Dermatology, Henry Ford Hospital, Detroit, Michigan, USA, Tel: +3139166964; E-mail: ihamzav1@hfhs.org

Received September 25, 2015; Accepted October 10, 2015; Published October 20, 2015

Citation: Mohammad T, Sommariva CE, Parks-Miller A, Hamzavi I (2015) Improvement of Vitiligo after Concurrent Treatment of Hypothyroidism: A Case Report. Pigmentary Disorders 2: 220. doi:10.4172/2376-0427.1000220

Copyright: (c) 2015 Mohammad T, et al. This is an open-access article distributed under the terms of the Creative Commons Attribution License, which permits unrestricted use, distribution, and reproduction in any medium, provided the original author and source are credited. 


\section{Discussion}

Hypothyroidism is the most common autoimmune disease associated with vitiligo [4]. This case report highlights the interplay between these two conditions, the importance of screening for autoimmune conditions, and the role concurrent treatment plays in achieving optimal repigmentation. A similar case was reported in the literature of an 18 year old female with vitiligo achieving repigmentation while on thyroid replacement, PUVA, oral corticosteroids, and an oral antioxidant [6]. The relationship between autoimmune hypothyroidism and vitiligo was investigated in a paper by Colucci et al. that looked at circulating auto-antibodies against thyroid hormones in vitiligo. These autoantibodies are more prevalent in people with vitiligo, which the authors suggest contributes to an inflammatory milieu, resulting in the formation of both autoimmune thyroid disease and vitiligo (Figure 2). The theory suggests that anti-thyroid hormone antibodies (Anti-TH $\mathrm{Ab}$ ) cross react with tyrosinase causing inactivation or impairment

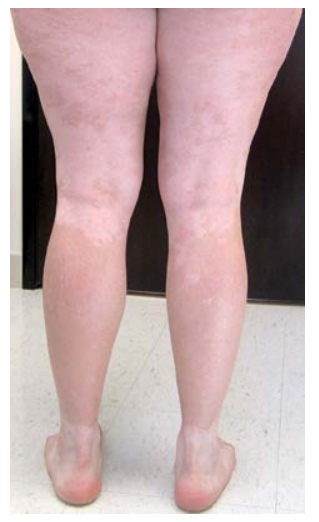

A

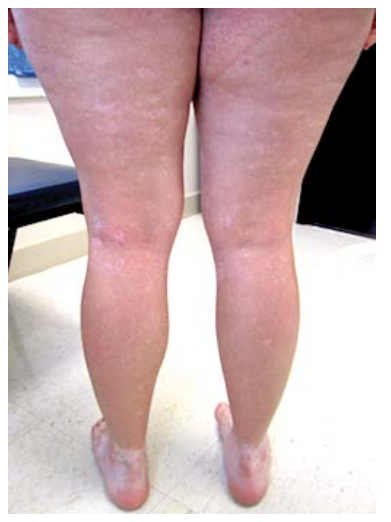

$\mathrm{B}$
Figure 2: 3 months of combined phototherapy (30 sessions of NBUVB phototherapy), oral corticosteroids, and thyroid replacement.

(2A) Legs at baseline showing $>75 \%$ depigmentation of upper legs (2B) Legs showing $>90 \%$ repigmentation of upper legs in a predominantly follicular pattern

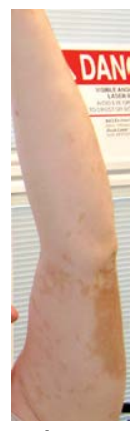

A

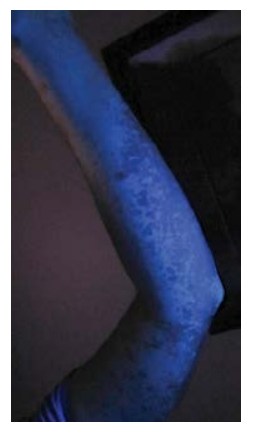

B

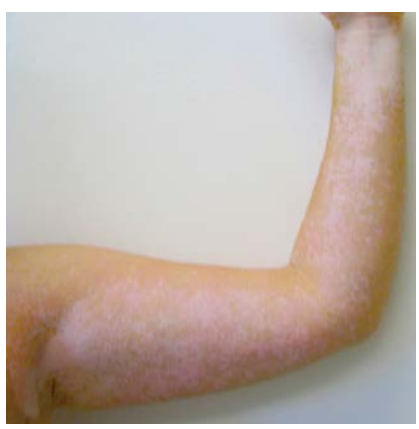

C
Figure 3: 4 months of treatment (40 sessions of NBUVB phototherapy) (3A) Ventral left arm at baseline showing $>75 \%$ depigmentation

(3B) Ventral left arm showing $>50 \%$ repigmentation in a predominantly follicular pattern

(3C) Back showing $>75 \%$ repigmentation in a predominantly follicular pattern and eventual loss of pigment. A potential cross reactivity with acetylcholinesterase, leading to increased acetylcholine levels in the skin, and subsequent inhibition of melanin production may also play a role. Increased levels of ROS also contribute as they initiate a cycle where increased ROS present in people with vitiligo causes thyroid damage and Anti-TH Ab production, which then leads to exacerbation of vitiligo by impairment of melanognesis [7]. This may be why treatment response improves once the inflammatory milieu is modulated with corticosteroids and phototherapy, and oral antioxidants are used to quench ROS (Figure 3). Currently, the role that thyroid hormone replacement plays in this process is unclear, but it is apparent that concurrent treatment of these two autoimmune conditions creates the optimal environment for repigmentation. However, additional studies are necessary to evaluate this mechanism further.

\section{References}

1. Daniel BS, Wittal R (2015) Vitiligo treatment update. Australas J Dermatol 56: 85-92.

2. Laddha NC, Dwivedi M, Mansuri MS, Gani AR, Ansarullah M, et al. (2013) Vitiligo: interplay between oxidative stress and immune system. Exp Dermatol 22: $245-250$.

3. Nunes DH, Esser LM (2011) Vitiligo epidemiological profile and the association with thyroid disease. An Bras Dermatol 86: 241-248.

4. Nejad SB, Qadim HH, Nazeman L, Fadaii R, Goldust M (2013) Frequency of autoimmune diseases in those suffering from vitiligo in comparison with normal population. Pak J Biol Sci 16: 570-574.

5. Gopal KV, Rao, Kumar YH (2014) Increased prevalence of thyroid dysfunction and diabetes mellitus in Indian vitiligo patients: A case-control study. Indian Dermatol Online J 5: 456-460.

6. Sehgal VN (2011) Vitiligo and alopecia areata associated with subclinical/ clinical hypothyroidism. Skinmed 9: 192-194.

7. Colucci R, Lotti F, Dragoni F, Arunachalam M, Lotti T, et al. (2014) High prevalence of circulating autoantibodies against thyroid hormones in vitiligo and correlation with clinical and historical parameters of patients. $\mathrm{Br} J$ Dermato 171: 786-798. 